\title{
TOPOLOGICAL PROOFS OF UNIQUENESS THEOREMS IN THE THEORY OF DIFFERENTIAL AND INTEGRAL EQUATIONS $\dagger$
}

\section{ERICH ROTHE}

It is known that, for a certain class of representations, the notion of the degree (Abbildungsgrad) can be transferred into Banach spaces and is useful for proving existence theorems for boundary value problems and integral equations. $\ddagger$ The same holds for the related notion of the order of a point with respect to the image of a sphere (Rothe [5]). It is the aim of the present paper to apply these notions to the proof of some uniqueness theorems. $\S$

Section 1 contains some uniqueness theorems for equations in Banach space. In $\$ 2$, application is made to a certain system of nonlinear integral equations for which the existence proof was given in [5].

1. Uniqueness theorems in abstract spaces. Let $E$ be a Banach space, $\|$ and let $\|\mathfrak{x}\|$ denote the norm of an element (point) $\mathfrak{x} \subset E$. Let $r$ be a positive number, $S$ the sphere $\|\mathfrak{x}\|=r$, and $V$ the "full" sphere $\|\mathfrak{x}\| \leqq r$. If then $\mathfrak{f}(\mathfrak{x})=\mathfrak{x}+\mathfrak{F}(\mathfrak{x})$ denotes a "representation with completely continuous translation," $\|$ we denote for any full sphere $V^{*} \subset V$ and its boundary $S^{*}$ the degree $\dagger \dagger$ in the point $\mathfrak{y}_{0} \subset E$, $\ddagger \ddagger$ with respect to the representation of $V^{*}$ given by $\mathfrak{f}$, by $\gamma\left(\mathfrak{f}, V^{*}, \mathfrak{y}_{0}\right)$, and likewise the order (see $[5, \S 2]$ ) of $\mathfrak{y}_{0}$ with respect to the image of $S^{*}$ by $u\left(\mathfrak{f}, S^{*}, \mathfrak{y}_{0}\right)$. If $\mathfrak{x}=\mathfrak{x}_{0}$ is an isolated solution of the equation $\mathfrak{f}(\mathfrak{x})=\mathfrak{y}_{0}$, then the number $\gamma\left(\mathfrak{f}, v, \mathfrak{y}_{0}\right)$ is the same for all full spheres $v$ with center $\mathfrak{x}_{0}$ which contain no other solution. $\S \S$ This number is called the index

$\dagger$ Presented to the Society, November 26, 1937.

$\ddagger$ Leray-Schauder [4]. The numbers in brackets refers to the list at the end of this paper.

$\S$ Considerations closely related to those of the present paper (especially of $\$ 1$ ) are to be found in $[3$, pp. 250,258$]$; cf. also the second footnote on page 610 of the present paper. Uniqueness proofs based on other topological ideas were given by R. Caccioppoli (see, for instance, Caccioppoli, Sugli elementi uniti delle trasformazioni funzionali, Rendiconti del Seminario Matematico, Padova, vol. 3 (1932), pp. 1-15) and G. Scorza Dragoni (see, for instance, Dragoni, Sui sistemi di equazioni integrali non lineari, Rendiconti del Seminario Matematico, Padova, vol. 7 (1936), pp. 1-35).

$\|$ For the definition of Banach space see $[1$, p. 53].

IT That is, the "translation" $\mathfrak{F}(\mathfrak{x})$ is unique and continuous, and the set of all points $\mathfrak{F}(\mathfrak{x})$ (with $\mathfrak{x} \subset V$ ) is compact.

$\dagger \dagger$ See [4, part I, §5].

$\ddagger \ddagger y_{0}$ is supposed not to lie on $f\left(S^{*}\right)$.

$\S \S$ See $[4$, part II, §8]. 
of the solution $\mathfrak{x}_{0}$ and will be denoted by $j\left(\mathfrak{f}, \mathfrak{x}_{0}\right)$, or simply by $j\left(\mathfrak{x}_{0}\right)$.

LEMma 1. For $0 \leqq t \leqq 1$ let $\mathfrak{x}=\mathfrak{x}_{0}(t)$ be a continuous curve in the interior of $V$; for each $t$ there is supposed to exist a full sphere $v$ with center $\mathfrak{x}_{0}(t)$, lying in the interior of $V$, and possessing this property: $\mathfrak{f}\left(\mathfrak{x}_{1}\right) \neq \mathfrak{f}\left(\mathfrak{x}_{2}\right)$ for any pair of different points $\mathfrak{x}_{1}, \mathfrak{x}_{2}$ lying in $v$. Then, the index $j(\mathfrak{f}, \mathfrak{x}(t))$ is independent of $t$.

Proof. Let $t^{*}$ be a fixed $t$ value, $\rho$ the radius of $v^{*}=v_{t^{*}}$, and $w^{*}$ the concentric full sphere with radius $\rho / 2$. On account of the Heine-Borel theorem, it will be sufficient to prove that if $\delta>0$ is chosen so that

$$
\left|t-t^{*}\right|<\delta
$$

implies $\mathfrak{x}_{0}(t)$ in the interior of $w^{*}$, then (1.1) implies also $j\left(\mathfrak{x}_{0}(t)\right)$ $=j\left(\mathfrak{x}_{0}\left(t^{*}\right)\right)$. To prove this, let $t^{* *}$ be a fixed $t$ value satisfying (1.1), and $w^{* *}$ the full sphere with center $\mathfrak{x}^{* *}=\mathfrak{x}_{0}\left(t^{* *}\right)$ and radius $\rho / 2$ so that

$$
\mathfrak{x}^{*}=\mathfrak{x}_{0}\left(t^{*}\right) \subset w^{* *} \subset v^{*} \text {. }
$$

Finally, let $w_{1}^{*}$ be a full sphere having $\mathfrak{r}^{*}$ as a center and lying in the intersection of $w^{*}$ and $w^{* *}$. Writing $\mathfrak{h}^{*}$ for $\mathfrak{f}\left(\mathfrak{x}^{*}\right)$, we see from (1.2) that in $w^{* *}$ (and in $\left.w_{1}^{*}\right) \mathfrak{x}=\mathfrak{x}^{*}$ is the only solution of the equation $\mathfrak{f}(\mathfrak{x})=\mathfrak{y}^{*}$. Therefore, by the definition of the index and by well known properties of the degree, $\uparrow$ it follows that, in obvious notation,

$$
j\left(\mathfrak{r}^{*}\right)=\gamma\left(\mathfrak{f}, w^{*}, \mathfrak{y}^{*}\right)=\gamma\left(\mathfrak{f}, w^{* *}, \mathfrak{y}^{*}\right) .
$$

We consider now the segment $g$ defined by

$$
\mathfrak{x}(\tau)=\mathfrak{x}^{*}+\tau\left(\mathfrak{x}^{* *}-\mathfrak{x}^{*}\right), \quad 0 \leqq \tau \leqq 1 .
$$

This segment connects the points $\mathfrak{x}^{*}$ and $\mathfrak{x}^{* *}$, is contained in the interior of $w^{* *}$, and therefore, according to (1.2), is also in $v^{*}$. Hence it follows from the hypothesis concerning $v^{*}$ that, if $x$ varies continuously along $g$ from $\mathfrak{x}^{*}$ to $\mathfrak{x}^{* *}, \mathfrak{f}(\mathfrak{x})$ is different from the image of the boundary of $w^{* *}$. Therefore, $\gamma\left(\mathfrak{f}, w^{* *}, \mathfrak{f}(\mathfrak{x}(\tau))\right)$ is defined and independent of $\tau$, and we have

$$
\gamma\left(\mathfrak{f}, w^{* *}, \mathfrak{f}\left(\mathfrak{x}^{*}\right)\right)=\gamma\left(\mathfrak{f}, w^{* *}, \mathfrak{f}\left(\mathfrak{x}^{* *}\right)\right)=j\left(\mathfrak{x}^{* *}\right) .
$$

Hence, from (1.3), $j\left(\mathfrak{x}^{* *}\right)=j\left(\mathfrak{x}^{*}\right)$, which was to be proved.

THEOREM 1. Let $y_{0}$ be a point of $E$ which is different from the image of $S$. Using the previous notations, we make the following assumptions:

(a) $u\left(S, \mathfrak{f}, \mathfrak{y}_{0}\right)= \pm 1$

(b) If the points $\mathfrak{x}^{\prime}, \mathfrak{x}^{\prime \prime}$ of $V$ are solutions of the equation $\mathfrak{f}(\mathfrak{x})=\mathfrak{y}_{0}$, they can be connected by a curve with the properties described in Lemma 1.

$\dagger$ See $[4$, part I, §1; and part I, §7]. 
Then the equation $\mathrm{f}(\mathfrak{x})=\mathfrak{y}_{0}$ has one and only one solution in $V$; the index of this solution is \pm 1 .

Proof. That there is at least one solution in $V$, follows immediately from (a). $\dagger$ As, on the other hand, all solutions are isolated, the number $n$ of different solutions must be finite. $\ddagger$ As the sum of the indices equals the order $\$$ and as, in accordance with Lemma 1, all indices have the same value $j$, it follows that $n j= \pm 1$, which proves our theorem.

LEMMA 2. Let $\mathfrak{l}_{t}(\mathfrak{x})$ be a representation with completely continuous translation for each value $t$ in the closed interval $[0,1]$. We make the following assumptions: if $\mathfrak{y}_{t}=\mathfrak{l}_{t}(\mathfrak{D})$, the solution $\mathfrak{x}=\mathfrak{D}$ of the equation $\mathfrak{l}_{t}(\mathfrak{x})=\mathfrak{y}_{t}$ is uniformly isolated, that is, there exists a full sphere $v$ with center $\mathfrak{o}$ and a radius independent of $t$ so that $v$ contains no other solution than $\mathfrak{x}=\mathrm{D}$; moreover, $\mathfrak{l}_{t}(x)$ is continuous in $t$, uniformly with respect to all $\mathfrak{x} \subset v$. Then the index $j\left(\mathfrak{l}_{t}, \mathfrak{D}\right)$ is independent of $t$.

Proof. Let $s$ be the boundary of $v$, and $t^{*}$ a fixed $t$-value. Let $\epsilon$ denote the positive distance between $\mathfrak{y}_{t^{*}}$ and $\mathfrak{l}_{t^{*}}(s)$, and $\delta$ a positive number so that

$$
\left\|\mathfrak{l}_{t}(\mathfrak{x})-\mathfrak{l}_{t^{*}}(\mathfrak{x})\right\|<\epsilon / 2
$$
$\mathfrak{x} \subset v$

for

$$
\left|t-t^{*}\right|<\delta
$$

On account of the Heine-Borel theorem, it will be sufficient to show that for such $t$

$$
j\left(\mathfrak{l}_{t}, \mathfrak{D}\right)=j\left(\mathfrak{l}_{t^{*}}, \mathfrak{D}\right) .
$$

To prove this, we notice that for all $\mathfrak{x} \subset s$ and for $t$ satisfying (1.5) the inequality

$$
\left\|\mathfrak{l}_{t}(\mathfrak{x})-\mathfrak{y}_{t^{*}}\right\| \geqq\left\|\mathfrak{l}_{t^{*}}(\mathfrak{x})-\mathfrak{y}_{t^{*}}\right\|-\left\|\mathfrak{l}_{t^{*}}(\mathfrak{x})-\mathfrak{l}_{t}(\mathfrak{x})\right\|>\epsilon-\epsilon / 2=\epsilon / 2
$$

holds because of (1.4) and the definition of $\epsilon$. Therefore

$$
\gamma\left(\mathfrak{Y}_{t^{*}}, v, \mathfrak{y}_{t^{*}}\right)=\gamma\left(\mathfrak{l}_{t}, v, \mathfrak{y}_{t^{*}}\right), \quad\left|t-t^{*}\right|<\delta .
$$

On the other hand, (1.4) shows that $\mathfrak{y}_{t}$ is contained in the interior of the full sphere with center $\mathfrak{y}_{t^{*}}$ and radius $\epsilon / 2$, while, according to $(1.7), \mathfrak{l}_{t}(s)$ lies in the exterior of this sphere for all $t$ satisfying (1.5).

$\dagger[5, \S 2$, Theorem 3$]$.

$\ddagger$ This follows easily from the fact that $\mathfrak{F}(\mathfrak{x})$ is completely continuous.

$\S$ See $[5, \S 3]$. 
Therefore

$$
\gamma\left(\mathfrak{l}_{t}, v, \mathfrak{y}_{t^{*}}\right)=\gamma\left(\mathfrak{l}_{t}, v, \mathfrak{y}_{t}\right) .
$$

As, by definition of the index, $j\left(\mathfrak{l}_{t^{*}}, \mathfrak{p}\right)=\gamma\left(\mathfrak{l}_{t^{*}}, v, \mathfrak{y}_{t^{*}}\right), j\left(\mathfrak{l}_{t}, \mathfrak{D}\right)=\gamma\left(\mathfrak{l}_{t}, v, \mathfrak{y}_{t}\right)$, (1.6) follows from (1.8) and $\left(1.8^{\prime}\right)$.

Before formulating the next lemma, it is convenient to give some definitions concerning "differentials" $: \dagger$

DeFINITION. The representation $\mathfrak{f}(\mathfrak{x})$ with completely continuous translation is said to be differentiable in the point $\mathfrak{x}_{0}$ when there exists a representation $\mathfrak{l}(\mathfrak{z})=\mathfrak{l}\left(\mathfrak{z}, \mathfrak{x}_{0}\right)$ with the following properties:

(a) $\mathfrak{R}(z)=\mathfrak{l}(z)-z$ is linear $\ddagger$ and completely continuous.

(b) $\lim _{\mathfrak{x} \rightarrow \mathfrak{x}_{0}}\left\|\mathfrak{f}(\mathfrak{x})-\mathfrak{f}\left(\mathfrak{x}_{0}\right)-\mathfrak{l}\left(\mathfrak{x}-\mathfrak{x}_{0}, \mathfrak{x}_{0}\right)\right\| /\left\|\mathfrak{x}-\mathfrak{x}_{0}\right\|=0$.

The differential $\mathfrak{l}$ is said to be nonsingular if the equation $\mathfrak{l}\left(\mathfrak{z}, \mathfrak{x}_{0}\right)=\mathfrak{D}$ has only the solution $z=\mathfrak{D}$. It is said to be continuous if it is continuous in $\mathfrak{x}_{0}$, uniformly for all $z$ of a bounded set.

Lemma 3. If $\mathfrak{f}(\mathfrak{x})$ possesses the nonsingular differential $\mathfrak{l}\left(\mathfrak{z}, \mathfrak{x}_{0}\right)$ in the point $\mathfrak{x}=\mathfrak{x}_{0}$ lying in the interior of $V$, then the solution $\mathfrak{x}$ of the equation $\mathfrak{f}(\mathfrak{x})=\mathfrak{f}\left(\mathfrak{x}_{0}\right)$ is isolated, and

$$
j\left(\mathfrak{f}(\mathfrak{x}), \mathfrak{x}_{0}\right)=j\left(\mathfrak{l}\left(\mathfrak{z}, \mathfrak{x}_{0}\right), \mathfrak{D}\right) . \S
$$

Proof. Let $S$ be the sphere $\left\|\mathfrak{x}-\mathfrak{x}_{0}\right\|=\rho_{1}$ where the positive number $\rho_{1}$ is so small that $S \subset V$. Since $\mathfrak{l}$ is nonsingular, $\mathfrak{l}\left(\mathfrak{x}-\mathfrak{x}_{0}, \mathfrak{x}_{0}\right)$ is different from $\mathfrak{D}$ for $\mathfrak{x} \subset S$; hence it follows $\|$ that for a certain positive number $d$

$$
\left\|\mathfrak{l}\left(\mathfrak{x}-\mathfrak{x}_{0}, \mathfrak{x}_{0}\right)\right\|>d, \quad \text { for }\left\|\mathfrak{x}-\mathfrak{x}_{0}\right\|=\rho_{1} .
$$

From this follows on account of the linearity of $\mathfrak{I}$ for any $\mathfrak{x} \neq \mathfrak{x}_{0}$

$$
\left\|\mathfrak{l}\left(\mathfrak{x}-\mathfrak{x}_{0}, \mathfrak{x}_{0}\right)\right\|=\frac{\left\|\mathfrak{x}-\mathfrak{x}_{0}\right\|}{\rho_{1}}\left\|\mathfrak{l}\left(\frac{\mathfrak{x}-\mathfrak{x}_{0}}{\left\|\mathfrak{x}-\mathfrak{x}_{0}\right\|} \rho_{1}, \mathfrak{x}_{0}\right)\right\|>\left\|\mathfrak{x}-\mathfrak{x}_{0}\right\| d / \rho_{1} .
$$

Let now $\rho_{2}$ be such a positive constant that for $0<\left\|\mathfrak{x}-\mathfrak{x}_{0}\right\| \leqq \rho_{2}$ the inequality

$$
\frac{\left\|\mathfrak{f}(\mathfrak{x})-\mathfrak{f}\left(\mathfrak{x}_{0}\right)-\mathfrak{l}\left(\mathfrak{x}-\mathfrak{x}_{0}, \mathfrak{x}_{0}\right)\right\|}{\left\|\mathfrak{x}-\mathfrak{x}_{0}\right\|}<\frac{d}{\rho_{1}}
$$

holds. This, together with (1.10), implies

$\dagger$ As to differentials in function spaces, see [2].

$\ddagger$ For the definition of linear transformations see, for instance, [1].

$\S$ This is known. Cf. [4, part II, §10]. For the sake of completeness, we give a complete proof.

\| See, for instance, $[5, \S 1,6]$. 


$$
\left\|\mathfrak{f}(\mathfrak{x})-\mathfrak{f}\left(\mathfrak{x}_{0}\right)-\mathfrak{l}\left(\mathfrak{x}-\mathfrak{x}_{0}, \mathfrak{x}_{0}\right)\right\|<\left\|\mathfrak{l}\left(\mathfrak{x}-\mathfrak{x}_{0}, \mathfrak{x}_{0}\right)\right\|
$$

so that

$$
\begin{array}{r}
\left\|\mathfrak{f}(\mathfrak{x})-\mathfrak{f}\left(\mathfrak{x}_{0}\right)\right\| \geqq\left\|\mathfrak{l}\left(\mathfrak{x}-\mathfrak{x}_{0}, \mathfrak{x}_{0}\right)\right\|-\left\|\mathfrak{f}(\mathfrak{x})-\mathfrak{f}\left(\mathfrak{x}_{0}\right)-\mathfrak{l}\left(\mathfrak{x}-\mathfrak{x}_{0}, \mathfrak{x}_{0}\right)\right\|>0 \\
\text { for } 0<\left\|\mathfrak{x}-\mathfrak{x}_{0}\right\| \leqq \rho_{2} .
\end{array}
$$

From this it follows that the full sphere $v$ with center $\mathfrak{x}_{0}$ and radius $\rho_{2}$ contains no solution of $\mathfrak{f}(\mathfrak{x})=\mathfrak{f}\left(\mathfrak{x}_{0}\right)$ except $\mathfrak{x}_{0}$. Hence, $\mathfrak{x}_{0}$ is isolated and we have, moreover,

$$
\begin{gathered}
j\left(\mathfrak{f}(\mathfrak{x}), \mathfrak{x}_{0}\right)=u\left(\mathfrak{f}(\mathfrak{x}), s, \mathfrak{f}\left(\mathfrak{x}_{0}\right)\right), \\
j\left(\mathfrak{l}\left(\mathfrak{x}-\mathfrak{x}_{0}, \mathfrak{x}_{0}\right), \mathfrak{x}_{0}\right)=u\left(\mathfrak{l}\left(\mathfrak{x}-\mathfrak{x}_{0}, \mathfrak{x}_{0}\right), s, \mathfrak{f}\left(\mathfrak{x}_{0}\right)\right)
\end{gathered}
$$

where, as usual, $s$ denotes the boundary of $v$ and $u$ the order. But a theorem proved in a previous paper* shows that (1.11) implies

$$
u\left(\mathfrak{f}(\mathfrak{x}), s, \mathfrak{f}\left(\mathfrak{x}_{0}\right)\right)=u\left(\mathfrak{l}\left(\mathfrak{x}-\mathfrak{x}_{0}, \mathfrak{x}_{0}\right), s, \mathfrak{f}\left(\mathfrak{x}_{0}\right)\right),
$$

so that the equality $j\left(\mathfrak{f}(\mathfrak{x}), \mathfrak{x}_{0}\right)=j\left(\mathfrak{l}\left(\mathfrak{x}-\mathfrak{x}_{0}, \mathfrak{x}_{0}\right), \mathfrak{x}_{0}\right)$ follows from (1.12). This proves (1.9) as it is easily seen that $j\left(\mathfrak{l}\left(\mathfrak{z}, \mathfrak{x}_{0}\right), \mathfrak{o}\right)=j\left(\mathfrak{l}\left(\mathfrak{x}-\mathfrak{x}_{0}, \mathfrak{x}_{0}\right), \mathfrak{x}_{0}\right)$.

Lemma 4. For $0 \leqq t \leqq 1$ let $\mathfrak{x}=\mathfrak{x}_{0}(t)$ be a continuous curve in the interior of $V$. In each point of this curve, $\mathfrak{f}(\mathfrak{x})$ is supposed to possess a nonsingular continuous differential $\mathfrak{l}\left(\mathfrak{z}, \mathfrak{x}_{0}(t)\right)$. We say that the index $j\left(\mathfrak{f}, \mathfrak{x}_{0}(t)\right)$ (which exists according to Lemma 3 ) is independent of $t . \dagger$

Proof. Upon putting $\mathfrak{l}_{t}(\mathfrak{z})=\mathfrak{l}\left(\mathfrak{z}, \mathfrak{x}_{0}(t)\right)$, it is seen from Lemma 3 , equation (1.9) that it is sufficient to prove the independence of $j\left(\mathfrak{l}_{t}(z), \mathfrak{D}\right)$ from $t$. This again follows from Lemma 2 since, by the assumptions made about $\mathfrak{l}_{t}$, the hypotheses of this lemma are fulfilled.

THEOREM 2. In our usual notation, $\ddagger$ let $\mathrm{y}_{0}$ be a point of $E$ not lying on the image of $S$. We make the following assumptions:

(a) $u\left(\mathfrak{f}, S, \mathfrak{y}_{0}\right)= \pm 1$.

(b) If the points $\mathfrak{x}^{\prime}$ and $\mathfrak{x}^{\prime \prime}$ of $V$ are solutions of the equation $\mathfrak{f}(\mathfrak{x})=\mathfrak{y}_{0}$, they can be connected by a curve with the properties described in Lemma 4.

Then the equation $f(x)=y_{0}$ has one and only one solution in $V$; the index of this solution is \pm 1 .

PROof. The proof of this theorem is obtained from the proof given for Theorem 1 by substituting the words "Lemma 4" for the words "Lemma 1."

* See $[5, \S 2$, Theorem 2a].

$\dagger$ Cf. [3, p. 250] where (without proof and explicit statement of the hypotheses) this independence of $t$ is used.

$\ddagger$ Compare the beginning of this section. 
2. Application to integral equations. Let $s, t$ denote points of an $m$-dimensional domain $B$. We consider the following system of nonlinear integral equations for the unknown functions $u_{1}(t), u_{2}(t), \cdots$, $u_{n}(t)$ :

$$
\begin{aligned}
u_{i}(s)+\int_{B} f_{i}\left(s, t, u_{1}(t), u_{2}(t), \cdots, u_{n}(t)\right) d t & =0, \\
i & =1,2, \cdots, n .
\end{aligned}
$$

The assumptions concerning the functions $f_{i}$ are the following:

(a) They are defined for

$$
s \subset B, \quad t \subset B, \quad \sum_{j=1}^{n}\left|u_{j}(t)\right| \leqq R,
$$

where $R$ is a positive number.

(b) For each system of continuous functions $u_{1}(t), \cdots, u_{n}(t)$, satisfying

$$
\sum_{j=1}^{n} \max \left|u_{j}(t)\right| \leqq R
$$

the integrals (2.1) exist.

(c) Moreover, these integrals are uniformly bounded and equicontinuous for the set of all systems of continuous functions $u_{1}(t), \cdots, u_{n}(t)$ satisfying $(2.2)$.

(d) There exist "dominant" functions $F_{j}\left(s, t, u_{1}, \cdots, u_{n}\right)$ with the following properties: they are defined for $s \subset B, t \subset B$, and all systems of nonnegative numbers $u_{1}, \cdots, u_{n}$ satisfying $\sum_{j=1}^{n} u_{j} \leqq R$; the integrals

$$
\int_{B} F_{i}\left(s, t, u_{1}(t), \cdots, u_{n}(t)\right) d t
$$

exist for all systems of nonnegative continuous functions $u_{1}(t), \cdots$, $u_{n}(t)$ satisfying $(2.2)$; moreover

(di) $\left|f_{i}\left(s, t, u_{1}, \cdots, u_{n}\right)\right| \leqq F_{i}\left(s, t,\left|u_{1}\right|, \cdots,\left|u_{n}\right|\right)$;

$\left(\mathrm{d}_{2}\right) F_{i}\left(s, t, u_{1}, \cdots, u_{n}\right) \leqq F\left(s, t, v_{1}, \cdots, v_{n}\right)$ for $0 \leqq u_{1} \leqq v_{1}, \cdots$, $0 \leqq u_{n} \leqq v_{n}$

$\left(\mathrm{d}_{3}\right)$ there exists a positive number $r \leqq R$ so that

$$
\sum_{j=1}^{n} \max \int_{B} F_{j}\left(s, t, r_{1}, r_{2}, \cdots, r_{n}\right) d t \leqq r
$$

holds for any system of nonnegative numbers $r_{1}, r_{2}, \cdots, r_{n}$ whose sum is $r$. 
Under these conditions, it was proved in $[5, \S 5]$ that the system (2.1) has at least one continuous solution $u_{1}(t), \cdots, u_{n}(t)$ with

$$
\sum_{j=1}^{n} \max \left|u_{j}\right| \leqq r .
$$

We prove now

THEOREM 3. The system (2.1) has only one continuous solution satisfying (2.4) if, in addition to (a)-( $\left.\mathrm{d}_{3}\right)$, the following conditions hold:

$\left(\mathrm{d}_{3}^{\prime}\right)$ Condition $\left(\mathrm{d}_{3}\right)$ remains true after the substitution of " $<$ " for "ฏ" in (2.3);

(e) for $s \subset B, t \subset B$, and all systems $u_{1}, \cdots, u_{n}$ with $\sum_{j=1}^{n}\left|u_{j}\right| \leqq r$ the differential quotients $\partial f_{i} / \partial u_{j}$ exist and are continuous functions of $\left(s, t, u_{1}, \cdots, u_{n}\right)$

(f) for any system of continuous functions $u_{1}(t), \cdots, u_{n}(t)$ satisfying (2.4), the system of linear integral equations

$$
z_{i}(s)+\int_{B} \sum_{j=1}^{n} \frac{\partial f_{i}\left(s, t, u_{1}(t), \cdots, u_{n}(t)\right)}{\partial u_{j}} z_{j}(t) d t=0, \quad i=1,2, \cdots, n,
$$

has only the solution $z_{1}=z_{2}=\cdots=z_{n}=0$.

Proof. Let $E$ be the Banach space whose points $x$ are the systems of continuous functions

$$
\mathfrak{x}=\left(u_{1}(t), u_{2}(t), \cdots, u_{n}(t)\right)
$$

and whose norm is given by

$$
\|\mathfrak{x}\|=\sum_{j=1}^{n} \max \left|u_{j}(t)\right|
$$

Let $\mathfrak{F}(\mathfrak{x})$ be the representation which makes correspond to the point $\mathfrak{x}$ the point

$$
\begin{aligned}
\mathfrak{F}(\mathfrak{x})=\left(\int_{B} f_{1}\left(s, t, u_{1}(t), \cdots, u_{n}(t)\right) d t,\right. & \cdots, \\
& \left.\int_{B} f_{n}\left(s, t, u_{1}(t), \cdots, u_{n}(t)\right) d t\right) .
\end{aligned}
$$

It was proved in a former paper* that, under the conditions (a)- $\left(\mathrm{d}_{3}\right)$, $\mathfrak{F}(\mathfrak{x})$ is completely continuous and that the inequality $\|\mathfrak{F}(\mathfrak{x})\| \leqq r$ holds

* See $[5, \S 5]$. 
for all points $x$ of the sphere $S$ defined by $\|\mathfrak{x}\|=r$. The proof given for this inequality shows that under the condition $\left(\mathrm{d}_{3}^{\prime}\right)$ even

$$
\|\mathfrak{F}(\mathfrak{x})\|<r, \quad \text { for }\|\mathfrak{x}\|=r,
$$

holds. That means that the translation of the points of $S$ caused by the representation $\mathfrak{f}(\mathfrak{x})=\mathfrak{x}+\mathfrak{F}(\mathfrak{x})$ is less than the radius of $S$, from which it follows* that the order $u(\mathfrak{f}, S, \mathfrak{p})$ equals 1 . Hence, hypothesis (a) of Theorem 2 is fulfilled.

Our theorem will be proved if we can show that hypothesis (b) of Theorem 2 is also fulfilled. For that purpose, we put

where

$$
\begin{aligned}
\mathfrak{x}_{0}= & \left(u_{01}(t), u_{02}(t), \cdots, u_{0 n}(t)\right), \\
\mathfrak{z}= & \left(z_{1}(t), z_{2}(t), \cdots, z_{n}(t)\right), \\
\mathfrak{l}\left(\mathfrak{z}, \mathfrak{x}_{0}\right)= & \left(z_{1}(s)+\int_{B} \sum_{j=1}^{n}\left[\frac{\partial f_{1}}{\partial u_{j}}\right]_{0} z_{j}(t) d t, \cdots, z_{n}(s)\right. \\
& \left.+\int_{B} \sum_{j=1}^{n}\left[\frac{\partial f_{n}}{\partial u_{j}}\right]_{0} z_{j}(t) d t\right),
\end{aligned}
$$

$$
\left[\frac{\partial f_{i}}{\partial u_{j}}\right]_{0}=\frac{\partial f_{i}\left(s, t, u_{01}(t), \cdots, u_{0 n}(t)\right)}{\partial u_{j}} .
$$

A simple application of the mean-value theorem shows then immediately that the conditions (e) and (f) imply that $\mathfrak{l}\left(\mathfrak{z}, \mathfrak{x}_{0}\right)$ is a continuous nonsingular differential of $\mathfrak{f}(\mathfrak{x})$ (in the sense of the definition given in $\S 1$ ) in each point of the full sphere $V$ defined by $\|\mathfrak{x}\| \leqq r$. As, on account of (2.5), all solutions of the equation $\mathfrak{f}(\mathfrak{x})=\mathfrak{x}+\mathfrak{F}(\mathfrak{x})=\mathfrak{v}$ lie in the interior of $V$, hypothesis (b) of Theorem 2 is indeed fulfilled.

\section{REFERENCES}

1. Banach, Théorie des Opérations Linéaires, Warsaw, 1932.

2. T. H. Hildebrandt and L. M. Graves, Implicit functions and their differentials in general analysis, Transactions of this Society, vol. 29 (1927), pp. 126-153.

3. J. Leray, Les problèmes de représentation conforme de Helmholtz; théories des sillages et des proues, Commentarii Matematici Helvetici, vol. 8 (1935-1936), pp. 149180, 250-263.

4. J. Leray and J. Schauder, Topologie et équations fonctionnelles, Annales Scientifique de l'École Normale Supérieure, vol. 51 (1934), pp. 45-78.

5. E. Rothe, Zur Theorie der topologischen Ordnung und der Vektorfelder in Banachschen Räumen, Compositio Mathematica, vol. 5 (1937), pp. 177-197.

William Penn College

* See $[5, \S 2$, Theorem $2 b]$. 